\title{
Fly Picture Measurement, un nouvel outil informatique pour l'étude des glossines
}

\author{
F. Borne ${ }^{1 *}$ L. Petiteau ${ }^{1}$ B. Geoffroy ${ }^{2}$ \\ S. de La Rocque ${ }^{3,4}$ D. Cuisance ${ }^{3}$
}

Mots-clés

Glossinidae - Glossina - Biométrie Logiciel - Mensuration - Aile.

\section{Résumé}

La biométrie intéresse l'entomologiste, d'une part comme élément de systématique, d'autre part comme indicateur des conditions de vie de l'insecte. Réalisée traditionnellement avec les moyens optiques classiques, elle est rendue plus rapide, fiable et précise grâce aux progrès de l'informatique (saisie et traitement informatisé des données). U n logiciel de mesure de la taille de segments remarquables et du niveau de gris moyen de l'aile a été conçu et appliqué à l'aile de glossine (Diptera : Glossinidae). Son intérêt pour l'entomologiste médical et vétérinaire est ici souligné.

\section{INTRO DUCTION}

La taille des insectes est un paramètre intéressant en entomologie médicale, car elle peut être reliée aux caractéristiques notamment abiotiques du milieu de vie et à la longévité des individus $(9,10)$. Dans le cas d'insectes vecteurs, la durée de vie est particulièrement importante à connaître puisque, compte tenu de la durée des cycles parasitaires, elle est un des éléments de base pour évaluer leur capacité vectorielle (15).

Chez les glossines, la taille des individus est appréciée à partir de mesures réalisées sur l'aile de l'insecte, organe dont les dimensions sont proportionnelles à celles du corps (4). Dans le genre Glossina, l'aile a une nervation constante et une cellule caractéristique en forme de hache, dont le côté coupant est fréquemment retenu pour les mesures (5). Celles-ci se réalisent sous le microscope, à l'aide d'un oculaire micrométrique. La méthode est évidemment lourde et fastidieuse. L'outil présenté ici permet de réaliser rapidement un ensemble de mesures de manière semi-automatique.

\footnotetext{
1. Cirad-amis, Unité de modélisation des plantes, BP 5035, 34032 Montpellier Cedex 1 Tél. : 0467548728 ; Fax : 0467548729 ; E-mail : frederic.borne@cirad.fr

2. Ird, Laboratoire de taxonomie et de chorologie des vecteurs, avenue du Val de Montferrand, 34032 Montpellier

3. Cirad-emvt, programme Econap, BP 5035, 34032 Montpellier Cedex 1

4. Cirdes, 01 BP 454, Bobo Dioulasso 01, Burkina Faso

* Auteur pour la correspondance
}

\section{PRESENTATION DU LOGICIEL FLY PICTURE MEASU REMENT (FPM )}

Sur l'image, l'utilisateur sélectionne avec sa souris huit points remarquables, situés à des intersections de nervures. Parmi ces points figurent notamment les cinq angles de la cellule en hache (figure 1). Il positionne également deux petits disques de $3 \mathrm{~mm}$ de diamètre, l'un sur une partie membraneuse de l'aile, l'autre en dehors de l'organe.

Le logiciel calcule alors la longueur en millimètres de trois segments, entre les points 6 et 8 (segment 1), 7 et 3 (segment 2), et 2 et 8 (segment 3 ). Il indique les rapports segment $1 /$ segment 2 et segment $1 /$ segment 3 , ainsi que la surface de la cellule en hache. Enfin, le niveau de gris moyen de l'aile est calculé par différence entre les valeurs obtenues dans les deux disques.

\section{MODE D'EM PLO I}

Les ailes des glossines sont individualisées et montées à sec entre lame et lamelle. La préparation est ensuite observée à la loupe binoculaire Olympus SZH au grossissement 75 (objectif : x 7,5 ; oculaire : x 10), sur laquelle est adaptée une caméra Canon XE1 reliée à un ordinateur. Il est également possible d'utiliser un scanneur si celui-ci a une résolution suffisante pour fournir une image supérieure à 500 x 500, afin de conserver une bonne précision géométrique. L'image est capturée sur l'écran et enregistrée (différents formats sont possibles : .tif, .bmp, .img). 


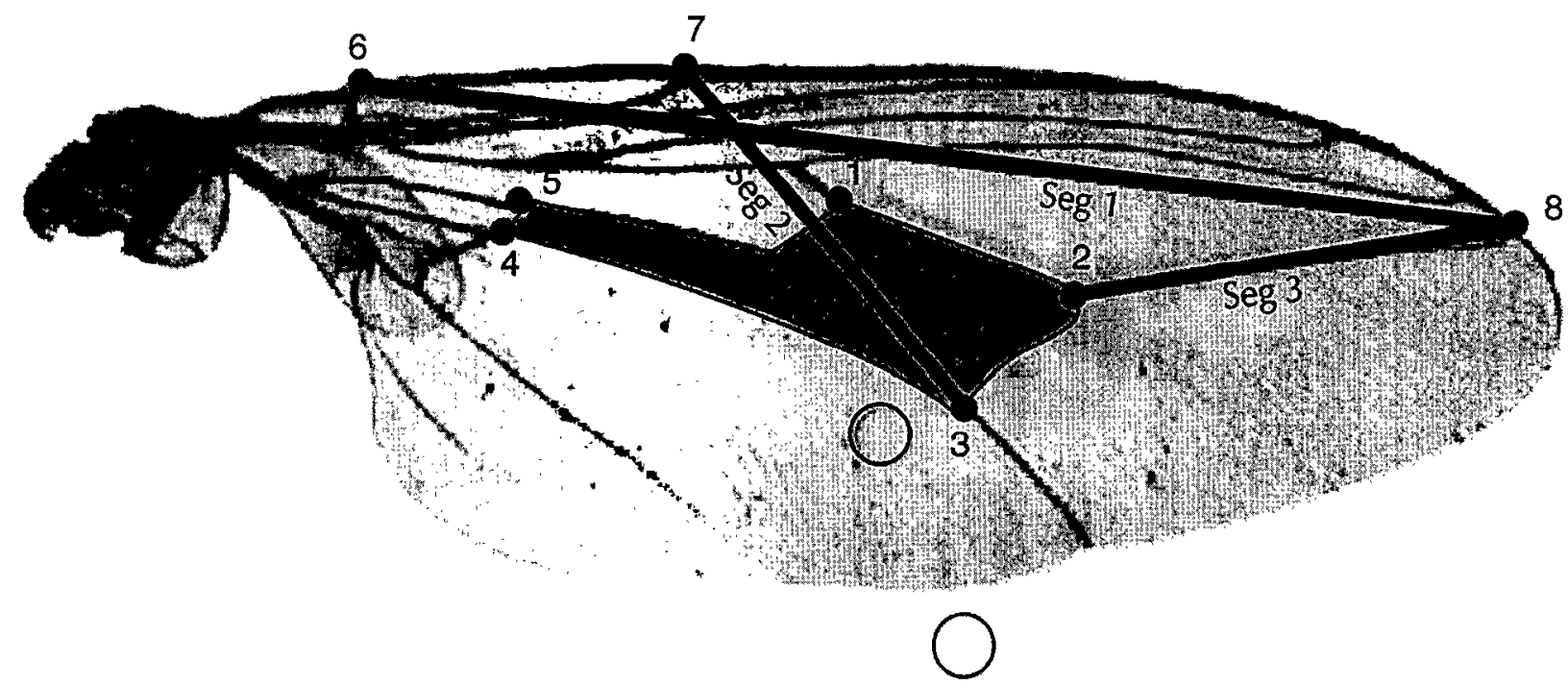

Figure 1 : localisation des points à saisir sur l'aile de glossine et paramètres calculés.

La simplicité a été recherchée dans l'utilisation de ce logiciel. Les principales étapes à suivre par l'utilisateur sont successivement : i) le chargement de l'image ; ii) un calibrage éventuel grâce à un étalon ; iii) la sélection des huit points de base.

Des outils ont été développés pour corriger de possibles imperfections de l'image : nettoyage de petites impuretés, amélioration de la netteté et de la lumière, renversement symétrique, agrandissement... Le logiciel est aussi capable de réaliser certaines des mesures, même en l'absence d'un des points remarquables. Il est également possible de demander des calculs supplémentaires sur l'image : surface de zones délimitées au curseur, longueur d'autres segments ou niveau de gris à l'intérieur de cercles de diamètres variables.

Les ailes de glossines « sauvages » présentent très occasionnellement de petites anomalies, comme des moignons de nervures surnumćraircs, en rapport, sclon ccrtains autcurs, avec les tcmpératures supportées dans le sol par la pupe de l'insecte $(12,20)$. Ces anomalies peuvent gêner le logiciel lorsqu'il délimite la cellule en hache et nécessitent alors une retouche de l'image.

Une aide en ligne est proposée. Elle est illustrée par des images d'écrans, afin de guider l'utilisateur, et enrichie de liens hypertextes, rendant son utilisation rapide et facile. Les résultats obtenus sont enregistrés dans un fichier de type tableur (.prn) ou en format texte (.txt) et peuvent alors être directement importés dans un logiciel de statistique.

Une démonstration du fonctionnement de ce logiciel est accessible sur Internet à l'adresse suivante :

$$
\text { http://www multimania .com/bornef/FPM/FPM.htm }
$$

\section{INTERET EN ENTOMOLOGIE}

FPM représente un apport important par rapport aux techniques utilisées jusqu'alors. Tout d'abord, ce logiciel donne accès à un ensemble de paramètres non mesurables visuellement ou avec une précision très incertaine. Ensuite, les résultats sont reproductibles et utilisables pour des analyses statistiques fiables. Enfin, le gain de temps est important puisque, avec un peu d'habitude, dcux à trois images peuvent être traitées en une minute. En revanche, la préparation des échantillons (montage entre lame et lamelle) est relativement laborieuse, surtout sur le terrain. Différents essais sont à réaliser pour définir un milieu de montage facile d'utilisation.

L'époque de l'entomologiste armé de son seul filet à papillon est bien révolue. L'entomologie bénéficie actuellement des technolo gies de pointe (3) et les études morphométriques, en particulier, bien qu'anciennes chez les insectes, connaissent un regain d'intérêt avec le développement d'outils tels que le logiciel présenté ici. Les applications des mesures morphométriques sont très vastes en entomologie (6). Elles offrent notamment de nouvelles possibilités pour l'identification d'espèces et de sous-espèces d'insectes $(2,14,19)$. Elles ont également été utilisées pour comparer des populations d'insectes à différentes échelles géographiques et apprécier par exemple leurs déplacements, que ce soit dans le cas de ravageurs tels que les acridiens (1) ou de vecteurs de maladies (11). Dans ce domainc de l'entomologic médicalc, ct notamment chez les glossines, mieux définir les populations de vecteurs (statut taxonomique, état physiologique, âge, histoire) permet d'apprécier leur aptitude à transmettre les parasites à un moment et en un lieu donnés $(7,8,9,13,16,18)$. Le logiciel FPM a, par exemple, été utilisé sur des populations « sauvages » de Glossina tachinoides et de Glossina palpalis gambiensis capturées dans la zone de Sidéradougou, au Burkina Faso. Les mesures obtenues sur les ailes sont suffisamment discriminantes pour différencier les deux espèces et le sexe des individus selon la forme, la taille ou la couleur de l'organe. Des variations ont également été observées selon la date de capture et les caractéristiques du milieu fréquenté (de La Rocquc et coll., en préparation), informations de première importance pour juger de l'état physiologique des populations. A ces caractérisations de plus en plus précises peuvent également être superposés des profils génétiques à̀ l'intérieur des espèces (17). Ces connaissances sont des éléments importants pour la compréhension du fonctionnement des divers «paysages épidémiologiques » et donc pour les décisions de lutte contre les maladies à transmission vectorielle qui restent dramatiquement d'actualité 
1. BLACKITH R.E., 1971. Morphometrics in acridology: A brief survey. Acrida, 1: 7-15.

2. BROWN K.R., 1979. Comparative wing morphometrics of some calyptrate Diptera. J. Aust. Entomol. Soc., 18: 289-303.

3. BRUHNES J., CUISANCE D., CUNY G., MANGUIN S., DE LA ROCQUE S., GEOFFROY B., 1998. Entomologie médicale : I'explosion technologique. Méd. trop., 58 : 15-20.

4. BURSELL E., 1960. The measurement of size in tsetse flies (Glossina). Bull. entomol. Res., 51: 33-37.

5. BUXTO N P.A., 1955. The natural history of tsetse flies. An account of the biology of the genus Glossina (Diptera). London, UK, London School of Hygiene and Tropical Medecine, 816 p. (Memoir N o. 10. Lewis H.K.)

6. DALY H.V., 1985. Insect morphometrics. Ann. Rev. Entomol., 30: 415-438.

7. DE DEKEN P., VAN DER BOSSCHE P., SANGARE M., GNANVI C., MISSANDA J.H., VAN HEES J., 1997. Effect of the life span of female Glossina palpalis gambiensis on the weight and size of its progeny. Med. vet. Entomol., 11: 95-101.

8. DE LA ROCQUE S., GEOFFROY B., CUISANCE D., 1996. Nouvelle approche pour l'estimation de l'âge des glossines par analyse d'image de I'aile. Revue Elev. Méd. vét. Pays trop., 49 : 46-48.

9. DEJARDIN J., MAILLOT L., 1964. Biométrie de la glossine : étude statistique des mensurations de l'aile dans diverses communautés (Glossina fuscipes quansensis, Pires). Revue Elev. Méd. vét. Pays trop., 17 : 97-102.

10. DRAN SFIELD R.D., BRIGHTWELL R., 1989. Problems of field testing theoritical models. A case study. Ann. Soc. belge Méd. trop., 69: 147-154.

\section{Summary}

Borne F., Petiteau L., Geoffroy B., de La Rocque S., Cuisance D. Fly Picture Measurement, a new software tool to study tsetse flies

Entomologists have shown interest in biometry, on one hand as a systematics tool, on the other hand because it gives indications on the living conditions of insects. Although it traditionally requires classical optical tools, it has now been made more reliable, precise and faster thanks to advances in computer science (data entry and processing). A software that measures the size of remarkable segments and the mean gray level of the wing was developed and applied to tsetse fly wings (Diptera: Glossinidae). Its applications for medical and veterinary entomologists are highlighted here.

Key words: Glossinidae - Glossina - Biometry - Computer software - Body measurement - Wing.
11. DUJARDIN J.P., MUNOZ M., CHAVEZ T., PONCE C., MORENO J. SCHOFIELD C.J., 1998. The origine of Rhodnius prolixus in Central America. Med. vet. Entomol., 12: 113-115.

12. GLASGOW J.P., 1960. Variation in the venation of Glossina Wiedermann (Diptera: Muscidae). Proc. R. Entomol. Soc. London, 35: 49-57.

13. GLASGOW J.P., 1961. Seasonal variation in size and colour, and daily changes in the distribution of Glossina pallidipes Aust. in the South Busoga Forest, U ganda. Bull. Entomol. Res., 52: 647-666.

14. LEBBE J., TORREZ M.E., VIGNES R., DEDET J.P., 1991. Wing morphology and species characterisation of sandflies. Parasitologia, $\mathbf{3 3}$ 365-372.

15. RODHAIN F., PEREZ C., 1985. Précis d'entomologie médicale et vétérinaire. Paris, France, Maloine S.A. Editeur, 458 p.

16. RO GERS D.J., RAND O LPH S.E., 1991. M ortality rate and population density of tsetse flies correlated with satellite imagery. Nature, $\mathbf{3 5 1}$ 739-741.

17. SO LANO P., DUVALLET G., DUMAS V., CUISANCE D., CUNY G. 1997. Microsatellite markers for genetic population studies in Glossina palpalis (Diptera: Glossinidae). Acta trop., 65: 175-180.

18. WALL R., 1990. O varian ageing of tsetse flies (Diptera: Glossinidae) Interspecific differences. Bull. entomol. Res., 80: 85-89.

19. WEEKS P.J.D., GAULD I.D., GASTON K.J., O'NEILL M.A., 1997. Automating the identification of insects: a new solution to an old problem. Bull. entomol. Res., 87: 203-211.

20. WELCH J.R., 1960. Variation in the venation of Glossina morsitans orientalis Vanderplanck (Diptera: Muscidae). Proc. R. Entomol. Soc. London, 35: 58-60.

Reçu le 14.1.99, accepté le 31.3.99

\section{Resumen}

Borne F., Petiteau L., Geoffroy B., de La Rocque S. Cuisance D. Fly Picture Measurement, un nuevo instrumento informático para el estudio de las glosinas

La biometría interesa al entomologista, como elemento de sistemática por un lado y como índice de las condiciones de vida del insecto por otro. Tradicionalmente llevada a cabo mediante los métodos ópticos clásicos, gracias al progreso de la informática (toma y tratamiento informático de datos), se vuelve más rápida, fiable y precisa. Se concibióun programa para medir el tamaño de los segmentos evidentes y del nivel medio de gris del ala, el cual se aplicó al ala de la glosina (Diptera: Glossinidae). Se señala el interés para el entomologista médico y veterinario.

Palabras clave: G lossinidae - Glossina - Biometria - Programa de ordenador - Medición del cuerpo - Ala. 